“C 2019 IEEE. Personal use of this material is permitted. Permission from IEEE must be obtained for all other uses, in any current or future media, including reprinting/republishing this material for advertising or promotional purposes, creating new collective works, for resale or redistribution to servers or lists, or reuse of any copyrighted component of this work in other works." 


\title{
Development of Robust Transparent Conformal Antennas Based on Conductive Mesh-Polymer Composite for Unobtrusive Wearable Applications
}

\author{
Abu Sadat Md. Sayem, Student Member, IEEE, Roy B. V. B. Simorangkir, Member, IEEE, \\ Karu P. Esselle, Fellow, IEEE, and Raheel M. Hashmi, Member, IEEE
}

\begin{abstract}
In this paper a detailed investigation of the realization of conformal wearable transparent antennas by integrating conductive mesh with polymer has been presented. The proposed realization method is much simpler and more cost-effective than the existing realization methods of transparent antennas, and the prototype fabricated from the selected composite materials is more flexible and robust in bending operations than other transparent antennas. In this paper the mechanical, electrical and optical characteristics of the proposed composite material have been investigated to analyze its suitability for transparent flexible antenna realization. For concept demonstration, a prototype of a dual-band antenna operating at $2.33-2.53 \mathrm{GHz}$ and $4.7-5.6 \mathrm{GHz}$ has been fabricated and tested. These frequencies cover both the instrument, scientific and measurement (ISM) and the wireless local area network (WLAN) bands. Full ground plane is utilized in the antenna design for on-body operations. The suitability of the antenna for wearable applications has been investigated by measuring its performance under physical deformation and testing its performance on phantom. Next, the RF performance of the antenna has been improved by using two layers of conductor to form the radiating element. Although transparency is slightly compromised, the double-layer element improves the gain and efficiency of the antenna.
\end{abstract}

Index Terms-Conformal antenna, human body, mesh, polydimethylsiloxane, robust, unconventional, wearable.

\section{INTRODUCTION}

A human body on which a wearable antenna operates is a unique environment, which imposes additional requirements on the antenna design and realization that are not applicable to other antennas [1], [2]. In the last decade, motivated by the increasing popularity of wearable wireless technologies, significant research efforts have been made to enhance antenna technology for applications in close proximity to a human body. Mechanical robustness and unobtrusiveness are of paramount importance in such applications, as the human body is a highly dynamic platform for antenna placement.

Recent efforts have focused on making wearable antennas visually unnoticeable and flexible to increase their aesthetic appeal and the comfort of the wearer [2], [3]. This is particularly important for continuous real-time body-centric communication systems such as the remote healthcare monitoring of older people, dementia care and security services. There

Manuscript received xxxx; revised xxxx; accepted xxxx. This research is supported by Commonwealth Government funded International Research Training Program (iRTP) Scholarship.

The authors are with the School of Engineering, Macquarie University, Sydney, NSW 2109, Australia (e-mail: abu-sadat-md.sayem@students.mq.edu.au). are several techniques to make an antenna unobtrusive. For example, embroidered antennas [4] can be directly integrated within clothing, and this is a good approach for achieving unobtrusiveness, but the performance of such antennas decreases after washing. Hiding wearable antennas inside clothing is another different solution but the performance of the antenna is affected by the layer of fabric upon it. In contrast to these approaches, making the antenna fully or nearly optically transparent is found to be the effective way to achieve the unobtrusiveness. Moreover, an optically transparent antenna may be used for other applications such as solar-cell panels, display devices and automobile windshields. In the last few years, a good amount of research has been conducted on transparent antennas [5]-[7], but unfortunately research efforts on transparent flexible antennas with robust performance are sparse due to the challenge of finding suitable materials.

Although some flexible transparent substrate materials such as Kapton polyimide, polydimethylsiloxane (PDMS) and polyethylene terephthalate are available, it is a challenge to find transparent flexible conductors. The transparent conductors commonly used for antenna fabrication are not suitable for wearable systems because of their undesirable mechanical properties. The commonly used transparent conductors are conductive films such as indium-tin-oxide (ITO) [8], [9], fluorine-doped tin oxide [10], aluminum-zinc-oxide [11] and silver-coated polyester film (AgHT) [12]. Among them, ITO is widely used as a transparent conductor but it is fragile and has low conductivity. In [13] heterogeneous gold nano layer was deposited on ITO film to improve the conductivity without sacrificing the transparency significantly. They used borosilicate glass as the substrate and the antenna attained a transparency level of $85 \%$, gain $3.5 \mathrm{dBi}$, efficiency $52 \%$ at 13 $\mathrm{GHz}$ and gain $0 \mathrm{dBi}$, efficiency $70 \%$ at $3 \mathrm{GHz}$. In [14] the flexibility and conductivity of ITO film were further improved by mixing with Zinc (Zn) and silver, and a monopole antenna was fabricated with this modified ITO film. This monopole antenna with a ground plane was integrated in wearable glass, where the average transparency was measured to be $81.1 \%$, the average efficiency was $40 \%$ and the peak gain was $4 \mathrm{dBi}$ at 2.4$2.5 \mathrm{GHz}$. This is another good approach for flexible transparent antenna fabrication. However, the high sheet resistance (4.99 $\Omega / \mathrm{sq}$ ) of the film contributes to antenna losses. Metallic mesh materials [15]-[17] have been used for fabricating transparent flexible antennas but their optical transparency is lower than that of ITO films. In [17] a tortuous copper micromesh was 
used for the conductive parts and PDMS as the substrate and encapsulation of the antenna. Tortuous copper micromesh is flexible and has an effective conductivity of $32800 \mathrm{~S} / \mathrm{cm}$. This PDMS encapsulated antenna was fabricated through the process of deposition with photolithography. The measured gain of the antenna was $-0.02 \mathrm{dBi}$ at $2.92 \mathrm{GHz}$, and its optical transmittances varied in the range of $32 \%-44 \%$ in the wavelength range of 400-800 $\mathrm{nm}$. Transparent antennas made from silver grid layers (AgGL) [18], [19] show good optical transparency but require the photolithographic wet etching process, which is not inexpensive enough for some low-cost applications.

In this paper, we present an efficient and inexpensive method of realizing transparent wearable antennas. The proposed method utilizes mesh conductive sheet as the antenna conductive parts and PDMS polymer as the substrate and encapsulation. Upon curing PDMS forms a strong integration with the conductive mesh, which produces a composite that is flexible, robust against physical deformations, optically transparent, protected from wet and humid conditions and is thus a promising candidate for flexible transparent antenna realization. In comparison to previously reported designs and realization methods of transparent antennas, the proposed method is much simpler and more cost-efficient, and the fabricated prototype is stable after repeated bending and twisting operations. As a demonstration a dual-band wearable patch antenna with a nearly transparent appearance is designed, fabricated and tested. The dual-band operation is achieved from a single rectangular patch antenna by utilizing slots and a tuning stub. Moreover, a full ground plane is utilized to sustain its performance on the human-body environment. The antenna performance is evaluated through on-phantom measurements and numerical calculation of the Specific Absorption Rate (SAR). In addition, the flexibility and durability of the antenna is evaluated by wrapping the antenna on a human-hand-shaped phantom and measuring its performance.

Finally a novel approach is investigated where two layers of mesh sheet are used to fabricate the antenna radiating element, which decreases the effective sheet resistance and thus improves the gain and efficiency of the antenna. All of these investigations and experiments have demonstrated the suitability of the new antennas and the effectiveness of the proposed fabrication method for easy and low-cost realization of robust, flexible, and transparent wearable antennas.

\section{MATERIAL SELECTION AND CHARACTERIZATION}

\section{A. Material selection}

The transparent antenna was realized by utilizing a composite material, PDMS-embedded conductive mesh. The conductive mesh was used as the antenna patch and ground and PDMS was used as the transparent substrate and protective coatings. To achieve optical transparency on the conductive parts, VeilShield from Less EMF Inc. was used which consists of woven mesh polyester threads coated with Zinc (Zn)blackened Nickel (Ni) over Copper. The threads intertwine with each other in a 1:1 ratio and at a $\pm 0.2 \mathrm{~mm}$ distance leading to a very thin and nearly transparent conductive sheet.

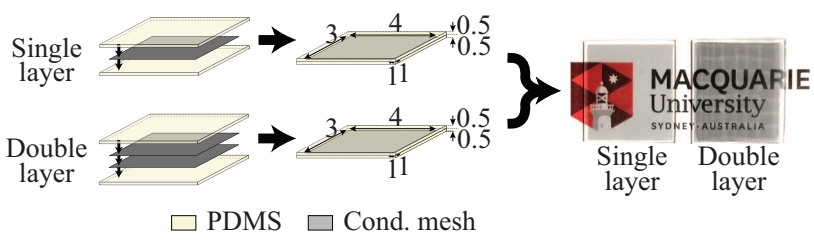

(a)

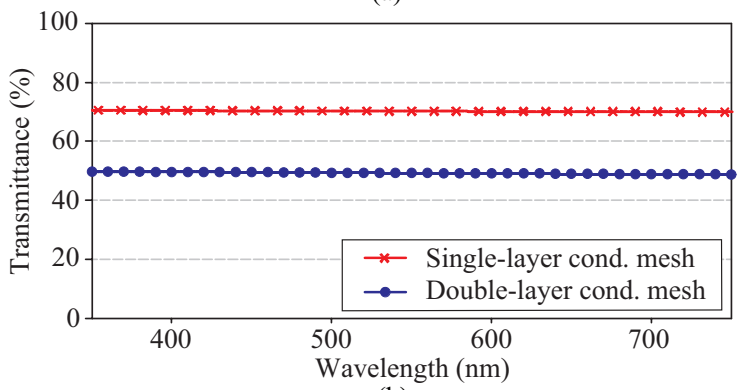

(b)

Fig. 1. (a) Test samples configurations (all dimensions of the geometries are in $\mathrm{mm}$ ) and photographs of the single-mesh-layer-PDMS and double-meshlayer-PDMS composites. The two samples were placed over a "Macquarie University" text and logo and the photograph indicates the difference in transparency between the two samples, (b) Measured transmittance.

According to the manufacturer's data sheet, it has a thickness of $57 \mu \mathrm{m}$ with $0.1 \Omega / \mathrm{sq}$ sheet resistance. The preparation of the PDMS solution was done by employing Dow Corning Sylgard 184 silicone elastomer kit with the base to curing agent mixing ratio of 10:1. The PDMS was found to have a relatively constant dielectric constant of 2.75 and an increasing loss tangent from 0.008 to 0.07 in the frequency range of 0.5 $\mathrm{GHz}$ to $10.6 \mathrm{GHz}$, based on our measurements with an Agilent 85070E Dielectric Kit. Cured PDMS is shock resistant, highly flexible, waterproof, chemically stable, and can withstand up to $400^{\circ} \mathrm{C}$ which increases the applicability of the antenna in harsh environments.

\section{B. Material characterization}

Prior to the design and realization of the transparent antenna, some investigations on the characteristics of the PDMSconductive-mesh composite were conducted. Firstly, its optical transparency was investigated by examining a rectangular sample of the composite material (see Fig. 1) under a Carry 5000 UV-vis-NIR spectrophotometer from Agilent. The optical transmittance was measured for both single-layer mesh and double-layer mesh. The latter measurement is relevant here because, to improve on the radiation performance of the first antenna, a double-layer radiator was used in the second antenna, as detailed later in Section V. The results illustrated in Fig. 1 show a good optical transmittance of approximately $70 \%$ for the single-layer-mesh-PDMS composite and approximately $50 \%$ for the double-layer-mesh-PDMS composite within the visible light spectrum from $350 \mathrm{~nm}$ to $750 \mathrm{~nm}$. It should be noted that the metal coating of VeilShield contains Zinc-blackened Nickel, which naturally absorbs visible light. Therefore, a higher optical transparency can potentially be achieved by modifying the metal composition of the thread coating.

Next, the morphology of the composite material was examined by observing a rectangular sample under a 


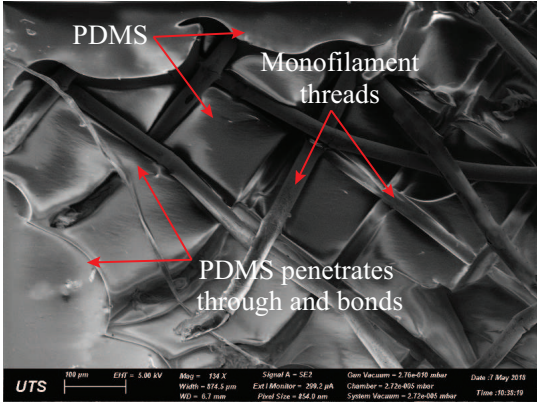

Fig. 2. SEM image of the PDMS-conductive-mesh composite.

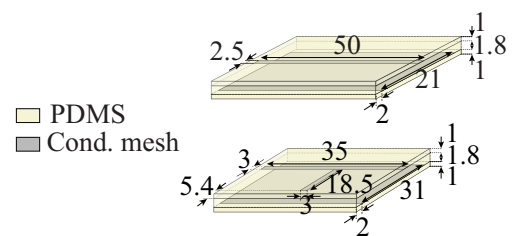

(a)

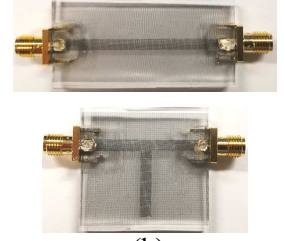

(b)
Fig. 3. The 50- $\Omega$ transmission line and the T-resonator made out of the PDMS-conductive-mesh composite: (a) configurations, (b) photographs of fabricated prototypes. (All dimensions are in $\mathrm{mm}$ ).

Zeiss Supra 55VP scanning electron microscope (SEM). Fig. 2 shows the top view of the sample with the top PDMS layer partly disrupted for a clear view of the interface between the fibres of the mesh and the PDMS. The result reveals a gridshaped structure made of the intertwining conductive threads placed on top of the PDMS layer. The pores of the mesh allow the percolation of PDMS solution, leading to bondings created between the PDMS layers on the top and bottom of the mesh, and hence sealing of the mesh upon curing. This explains how the attachment between the two classes of material, which is inherently weak [20], has been achieved. The strong attachment between the mesh and PDMS is the reason for the robustness of the composite under repeated mechanical deformations. The mechanical robustness achieved was also found to be better than the similar composite material reported in [21]. Due to the lower porosity of the fabrics used in [21], detachment of the fabrics from the PDMS under repetitive harsh deformations is still likely to happen. So, the added advantage of our proposed composite is the physical robustness besides optical transparency.

The electrical properties of the composite were also characterized by designing and fabricating samples of a $50-\Omega$ transmission line and a T-resonator (see Fig. 3). In the simulations using CST Microwave Studio 2017, initially the conductive mesh sheet was modeled as a box having thickness and sheet resistance as specified by the manufacturer. However, for more accurate results, the loss contributed by the PDMS penetrating into the mesh was considered. This was done by adjusting the sheet-resistance value of the mesh until the best match was reached between the simulated and measured S-parameters of the transmission line [22]. For a better estimation, the same process was repeated with the T-resonator sample [23]. From these investigations we found that an approximate sheet resistance of nearly $0.7 \Omega / \mathrm{sq}$ was required to model the mesh

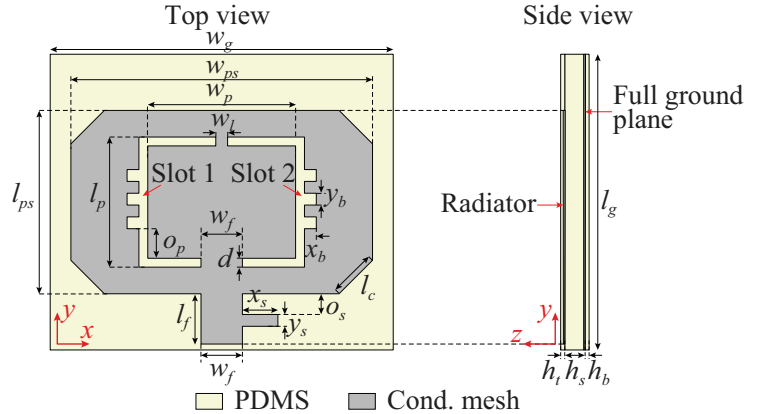

Fig. 4. Configuration of the new transparent dual-band antenna.

TABLE I

DIMENSIONS OF THE NEW ANTENNA

\begin{tabular}{c|c|c}
\hline Parameter & Description & Value $(\mathrm{mm})$ \\
\hline \hline$l_{p s}$ & Length of the outer patch & 31 \\
$w_{p s}$ & Width of the outer patch & 51 \\
$l_{p}$ & Length of the inner patch & 19 \\
$w_{p}$ & Width of the inner patch & 25 \\
$l_{f}$ & Length of the feed-line & 8.5 \\
$w_{f}$ & Width of the feed-line & 7 \\
$l_{g}$ & Length of the ground plane & 50 \\
$w_{g}$ & Width of the ground plane & 58 \\
$d$ & Gap between inner patch and outer patch & 1.5 \\
$l_{c}$ & Length of the corner cut & 8 \\
$w_{l}$ & Width of the shorting line & 2 \\
$x_{s}$ & Length of the stub & 6 \\
$y_{s}$ & Width of the stub & 2 \\
$o_{s}$ & Distance from patch to stub & 2 \\
$x_{b}$ & Length of the slot & 2 \\
$y_{b}$ & Width of the slot & 2 \\
$o_{p}$ & Distance from inner patch to slot & 5 \\
$h_{t}$ & Thickness of the top cover & 0.76 \\
$h_{b}$ & Thickness of the bottom cover & 0.76 \\
$h_{s}$ & Thickness of the substrate layer & 3 \\
\hline \multicolumn{2}{|}{}
\end{tabular}

with PDMS penetration. This value was later used in the antenna design phase.

\section{TRAnSPARENT FLEXIBLE ANTENNA REALIZATION}

\section{A. Antenna design}

Using the selected materials we designed a dual-band antenna operating at $2.33-2.53 \mathrm{GHz}$ (first band) and $4.7-5.6 \mathrm{GHz}$ (second band). The configuration of the antenna is shown in Fig. 4. Table I shows the dimensions of the antenna.

The antenna design is based on a rectangular patch antenna operating at its fundamental mode. Two slots (slot 1 and 2) divide the patch into inner and outer patches and are used to tune each band independently. As shown in Figs. 5(a) and (b), the lower resonance frequency can be tuned by varying the dimensions of the outer section (e.g., $l_{p s}$ ), while the tuning of the upper resonance frequency can be done by modifying the dimensions of the inner section (e.g., $l_{p}$ ). Some modifications such as cutting the corners of the outer section and six extra slots were introduced to slightly increase the current path lengths and current density to improve the gain. Moreover, the other benefit of cutting these parts of the patch is to increase the light transmittance of the antenna which is one of our design goals. A stub was introduced to improve matching in the upper band. The final design of the antenna, obtained with the aid of CST Microwave Studio 2017 full-wave simulator, is shown in Fig. 4. 


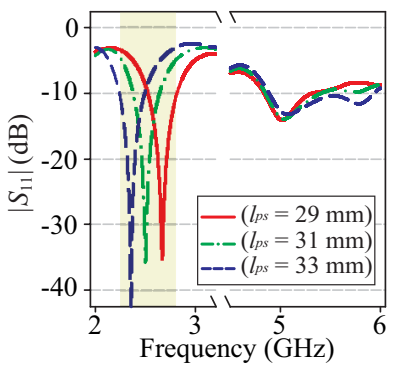

(a)

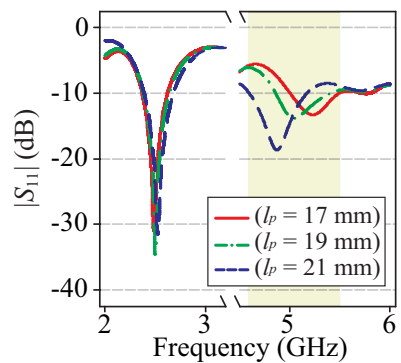

(b)
Fig. 5. Predicted magnitude of the input reflection coefficient of the antenna for varying (a) $l_{p s}$ and (b) $l_{p}$. All other parameters are constant.

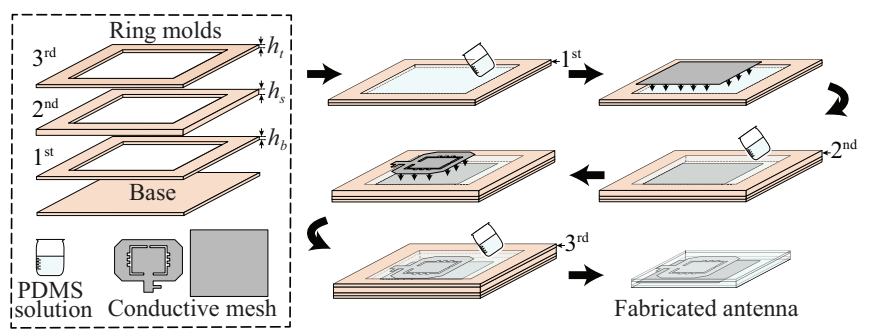

Fig. 6. Step-by-step fabrication process of the antenna.

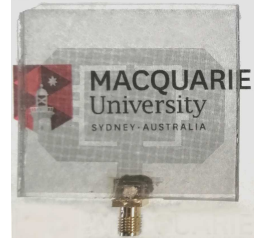

Top view

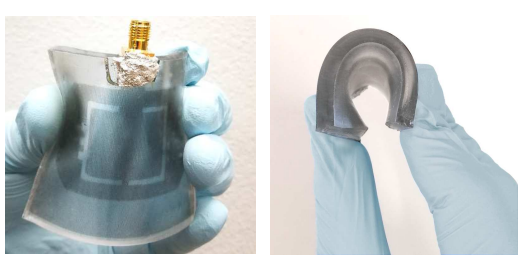

Views of bent antenna
Fig. 7. Photographs of the fabricated flexible transparent antenna. On the left photo, the Macquarie University logo and name, printed on paper, can be seen through the antenna.

\section{B. Prototype Fabrication}

The antenna fabrication process was conducted through a layer-by-layer assembly process as shown in Fig. 6. The three layers of the antenna were constructed one after another starting from the bottom protective layer. Three rectangularshaped molds having dimensions matched to the design given in Fig. 4 were used. The patch and ground plane were made by cutting the mesh sheet manually with a sharp razor blade. Then sticky tape was attached at the feed-line and the ground where an SMA connector was to be connected. This was to resist the percolation of the PDMS into the mesh at those particular sections, thus facilitating the attachment of the connector described in the next paragraph.

Firstly the bottom protective layer was fabricated by pouring uncured PDMS on the first mold attached to a base. The uncured PDMS was degassed in a vacuum desiccator by applying -80 kPA pressure to completely remove air bubbles and then cured at $75^{\circ} \mathrm{C}$ in an oven for 1 hour. After curing this layer, the ground plane, including the tape attached earlier at the SMA connecting point, was attached on top of it with a small amount of uncured PDMS and then cured at $75^{\circ} \mathrm{C}$ for 20 minutes. At the next stage, the second mold was attached to

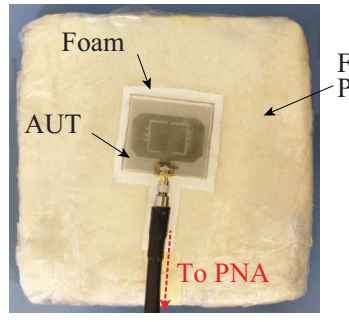

(a)
Flat

Phantom

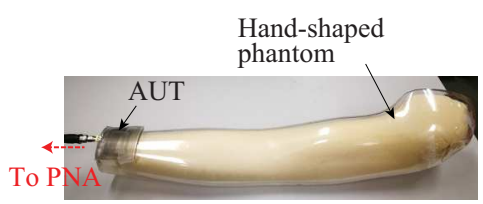

(b)
Fig. 8. On-phantom $\left|S_{11}\right|$ measurement set-ups: (a) the antenna is mounted on top of the flat phantom, (b) the antenna is bent around the wrist of the human-hand-shaped phantom.

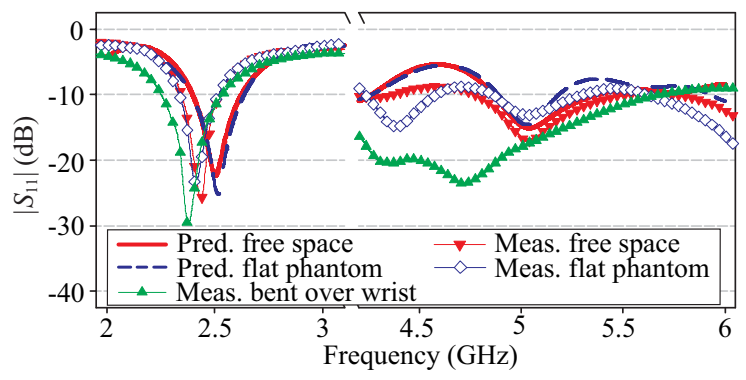

Fig. 9. Predicted and measured $\left|S_{11}\right|$ of the antenna in free space, when placed on a flat phantom and when bent over the wrist of the phantom.

the first mold by using silicone sealant and then the substrate layer was made by following the same steps as in fabricating the bottom protective layer. After finishing the substrate layer the patch was attached to it with small amount of PDMS in the same way that the ground plane was attached to the bottom layer. The patch was adhered on the substrate by keeping the tape attached at the feed line where the pin of the SMA was to be connected. Then the third mold was attached to the second mold and the top PDMS protective layer was made. After final curing, the antenna was peeled off from the molds.

For measurement purposes, the SMA connector was connected to the feed-line and ground with silver epoxy after peeling off slightly the top and bottom PDMS protective layers around the feed line and ground. Without the sticky tape applied earlier, it is impossible to peel off any portion of the PDMS coatings after curing as they bond very well through the pores of the mesh. The silver epoxy was cured in the oven at $75^{\circ} \mathrm{C}$ for 2 hours. Fig. 7 shows the fabricated prototype of the antenna. It can be noticed that the text and the logo underneath the antenna can be clearly seen through the antenna prototype, verifying its transparency and hence unobtrusiveness, and the views of the bent antenna reveal the flexibility and robustness. As can be seen in Fig. 7 the transparency is less in the patch area, simply because of the existence of another layer of conductive mesh underneath the patch utilized as the full ground plane in this design example. It can therefore be implied that more transparency in the antenna can be achieved through a coplanar design, at the expense of ineffectiveness of the electromagnetic shielding towards human body. 


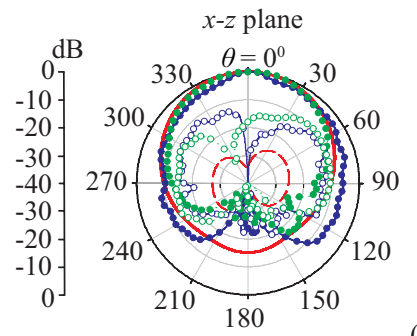

(a)
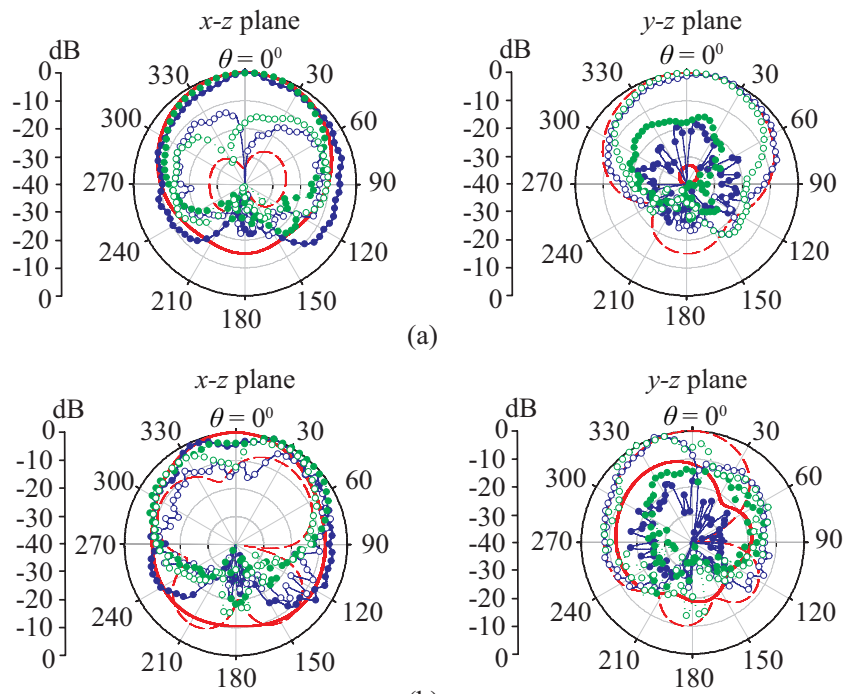

(b)

\begin{tabular}{|c|c|}
\hline $\begin{array}{l}\text { - Pred. } \mathrm{E}_{\phi} \text { free } \\
\ldots \text { Meas. } \mathrm{E}_{\phi} \text { fre } \\
\cdots \text { - Meas. } \mathrm{E}_{\phi} \text { fla }\end{array}$ & $\begin{array}{l}\text { - Pred. } \mathrm{E}_{\theta} \text { free space } \\
\ldots \text { - Meas. } \mathrm{E}_{\theta} \text { free space } \\
\ldots \circ \text { Meas. } \mathrm{E}_{\theta} \text { flat phantom }\end{array}$ \\
\hline
\end{tabular}

Fig. 10. Predicted and measured normalized radiation patterns of the antenna in free space and on the flat phantom: (a) $2.5 \mathrm{GHz}$, (b) $5 \mathrm{GHz}$.

\section{TRANSPARENT ANTENNA PERFORMANCE}

The RF performance of the antenna was characterized in both free space and on a human-body environment. For the latter, two ultra-wideband (UWB) homogeneous semisolid phantoms (namely a flat phantom with dimensions of $200 \mathrm{~mm}$ $\times 200 \mathrm{~mm} \times 47 \mathrm{~mm}$ and a human-hand-shaped phantom), as shown in Fig. 8, were used. The phantoms were fabricated by following the procedure mentioned in [24] using deionized water $(85.15 \%)$, agar (2.64\%), polyethylene powder (PEP) $(10.52 \%)$, sodium chloride $(0.18 \%), \mathrm{TX}-151(1.46 \%)$ and sodium dehydroacetate $(0.05 \%)$. The electrical properties of the phantoms, measured using an Agilent 85070E Dielectric Probe Kit, mimic quite well the electrical properties of human muscle over a very wide frequency range that covers both the operating bands of this antenna. To emulate the placement of the antenna on a human body with clothing, a $5 \mathrm{~mm}$ gap was maintained between the antenna and the phantom during the measurements by means of a foam slab.

Fig. 9 compares the measured $\left|S_{11}\right|$ of the antenna with the results predicted from the simulations in free space and on a flat phantom, and shows reasonably good agreement. The discrepancies in the results are attributed to fabrication errors incurred when cutting the mesh sheet and variations in the electrical properties of the fabricated phantoms. The former includes a limited precision of manual cutting and the tendency of the edge of the mesh sheet to fray upon cutting. In free space, a measured 10-dB return-loss bandwidth of $200 \mathrm{MHz}$ (from $2.33 \mathrm{GHz}$ to $2.53 \mathrm{GHz}$ ) was achieved in the lower band and $900 \mathrm{MHz}$ (from $4.7 \mathrm{GHz}$ to $5.6 \mathrm{GHz}$ ) in the upper band. When the antenna was measured on the flat phantom, measured 10-dB return-loss bandwidths of $170 \mathrm{MHz}$ (from $2.34 \mathrm{GHz}$ to $2.51 \mathrm{GHz}$ ) and $700 \mathrm{MHz}$ (from $4.8 \mathrm{GHz}$ to $5.5 \mathrm{GHz}$ ) were obtained in the lower and higher bands, respectively. From the comparison of the measured resonance
TABLE II

Summary of the FreE-SPACE AND ON-Phantom Performance of THE ANTENNA

\begin{tabular}{c|c|c|c|c|c|c}
\hline \multirow{2}{*}{ Band } & \multicolumn{2}{|c|}{ Bandwidth (MHz) } & \multicolumn{2}{c|}{ Peak Gain (dBi) } & \multicolumn{2}{c}{ Max. Eff. (\%) } \\
\cline { 2 - 7 } & FS & OP & FS & OP & FS & OP \\
\hline \hline Lower & 200 & 170 & 2.2 & 1.7 & 37 & 32 \\
Higher & 900 & 700 & 3.02 & 2.27 & 44 & 36 \\
\hline
\end{tabular}

Note: FS: Free Space, OP: On Phantom

positions and bandwidths of the antenna in free-space and on-phantom, it is identified that the antenna resonances and bandwidths are not affected significantly by the phantom, confirming the isolation provided by the full ground plane underneath the radiator.

Fig. 9 also illustrates the effect of physical deformation on the antenna performance. The antenna was wrapped around the wrist of the hand-shaped phantom having an extreme bending radius of $28 \mathrm{~mm}$ as shown in Fig. 8(b). From Fig. 9 it can be seen that the resonance frequencies shift downward under bending, which can be understood because the current path elongates under bending, decreasing the resonance frequencies. However, more importantly, the attachment of the mesh and the PDMS remains stable even after repetitive bending operations, and hence so does the antenna performance.

The far-field radiation patterns of the flat antenna were measured using the NSI700S-50 spherical near-field antenna range at the Australian Antenna Measurement Facility in CSIRO, Marsfield, Sydney, Australia in both free-space and on-phantom environments. The flat phantom was used for the measurements. The predicted and measured patterns at 2.5 and $5 \mathrm{GHz}$ shown in Fig. 10 indicate that the antenna radiates in the broadside direction, with a good agreement with predicted results. Moreover, the comparison of free-space and on-phantom results proves the further stability of the antenna operation in a human-body environment, which is a benefit of using a full ground plane. The measured peak gain of the antenna in free space is $2.2 \mathrm{dBi}$ and $3.02 \mathrm{dBi}$ at the lower band and upper band, respectively, whereas on the phantom these values are $1.7 \mathrm{dBi}$ and $2.27 \mathrm{dBi}$, respectively. The measured maximum attainable efficiency of the antenna in free space is $37 \%$ in the lower band and $44 \%$ in the upper band, whereas on the phantom the efficiencies are $32 \%$ and $36 \%$ at the lower band and upper band respectively. The measured performance of the antenna in free-space and on-phantom are summarized in Table II.

Finally, the SAR level of the antenna was investigated numerically to determine the effect of its radiation on a human body. To determine the SAR level, CST Microwave Studio 2017 was used, with the antenna placed $5 \mathrm{~mm}$ above a 200 $\mathrm{mm} \times 200 \mathrm{~mm} \times 47 \mathrm{~mm}$ human-muscle-equivalent phantom. The SAR values were calculated by applying $0.1 \mathrm{~W}$ of input power and averaged over $10 \mathrm{~g}$ of tissue. The calculated SAR distributions at 2.5 and $5 \mathrm{GHz}$ are given in Fig. 11, showing maximum values of $0.0406 \mathrm{~W} / \mathrm{kg}$ and $0.167 \mathrm{~W} / \mathrm{kg}$ at these frequencies respectively. These are well below the SAR limit of $2 \mathrm{~W} / \mathrm{kg}$ specified in the IEEE C95.1-2005 standard. The results also imply that the input power level can be increased 


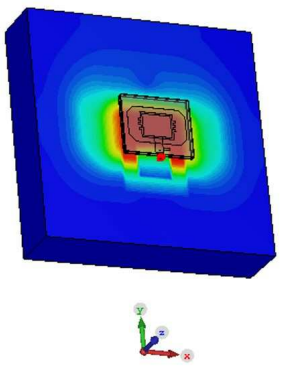

(a)
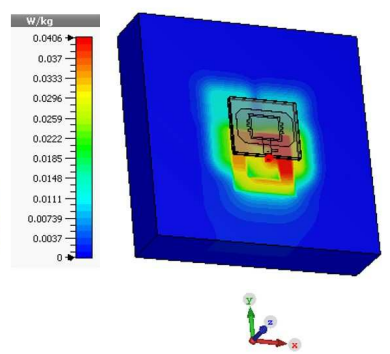

(b)
Fig. 11. Predicted SAR distribution in human-muscle-equivalent flat phantom: (a) $2.5 \mathrm{GHz}$, (b) $5 \mathrm{GHz}$.

up to $1 \mathrm{~W}$ without exceeding the IEEE SAR specification.

\section{PERformance EnHANCEMENT By USing DOUBLE-LAYER RADIATOR}

Although the investigated antenna demonstrates the effectiveness of the proposed method, its gain and efficiency are lower than those of typical rigid transparent antennas. One reason is due to the conductivity imperfection of the conductive mesh. The very porous nature of the selected transparent conductor, Veilshield, leads to discontinuity of the current flow leading to higher sheet resistance. Another major cause of the antenna's low efficiency is the loss contributed by the PDMS used to compose the substrate and protective layers. PDMS is notorious for its lossy characteristic, especially at high frequencies. Based on our measurements, it shows loss tangents of 0.021 and 0.047 at our respective target frequencies of $2.5 \mathrm{GHz}$ and $5 \mathrm{GHz}$, leading to approximately $34-39.7 \%$ energy loss in the antenna. With such drawback, the utilization of PDMS nevertheless has successfully provided the transparency as well as the desired superior mechanical robustness (i.e., flexibility, versatility, resilience to extreme environments) which can't be achieved by other rigid transparent materials such as glass. It is also very easy to fabricate and cost efficient.

To improve the gain and efficiency of the antenna, we investigated a novel approach of using a double-mesh layer instead of a single-mesh layer (see Fig. 1(a)) for constructing the patch layer of the antenna. By doing so, the discontinuities in the mesh can be minimized and therefore the effective sheet resistance can be decreased. Moreover, a stronger connection between the antenna feed line and SMA connector can also be achieved as the mesh surface becomes more continuous and hence attached better with silver epoxy.

The fabrication process of the double-layer radiator antenna was similar to that of the single-layer radiator antenna, except for the construction of the patch. After the attachment of the conductive mesh forming the patch layer, another conductive mesh, having identical geometry to the first one, was attached with a small amount of uncured PDMS. Extra care was taken in aligning the second mesh right on top of the first one to avoid any change in the overall dimensions of the antenna patch. The antenna was then cured in the oven at $75^{\circ} \mathrm{C}$ for 30 minutes to bond the two patches together and to the substrate PDMS layer. The remaining steps were done following the procedure outlined previously. Fig. 12 shows the fabricated

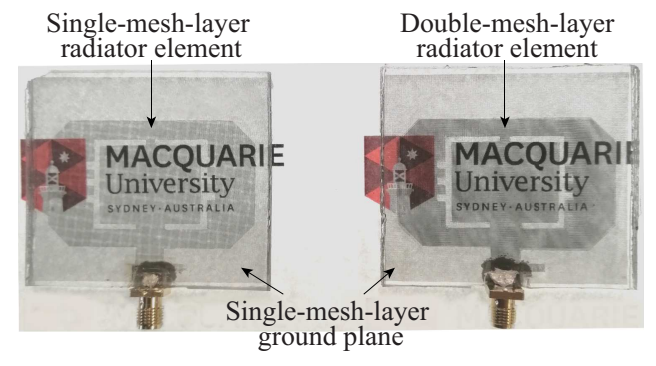

Fig. 12. The two antenna prototypes side-by-side.

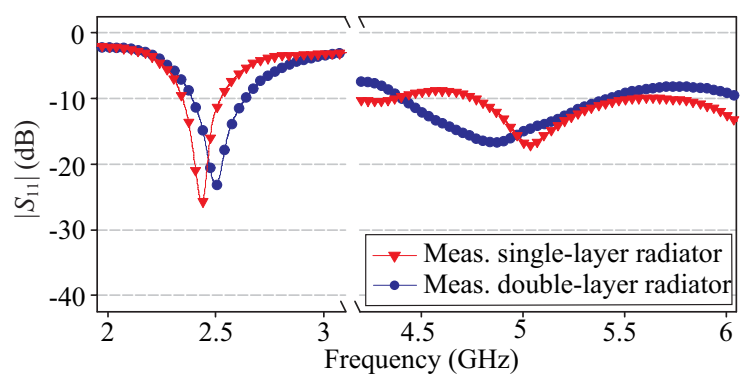

Fig. 13. Measured $\left|S_{11}\right|$ of the double-layer radiator antenna along with that of the single-layer radiator antenna.
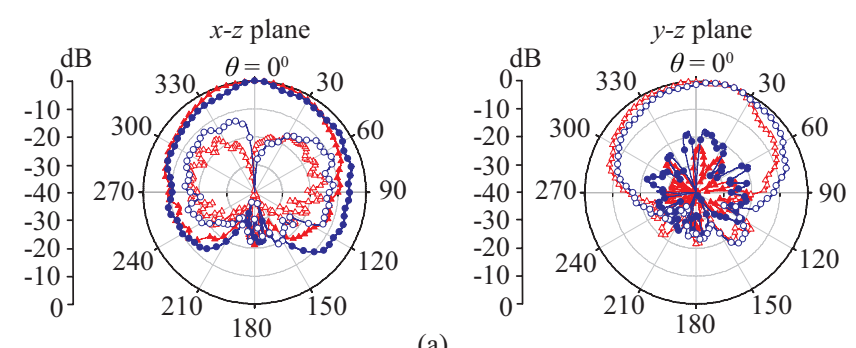

(a)
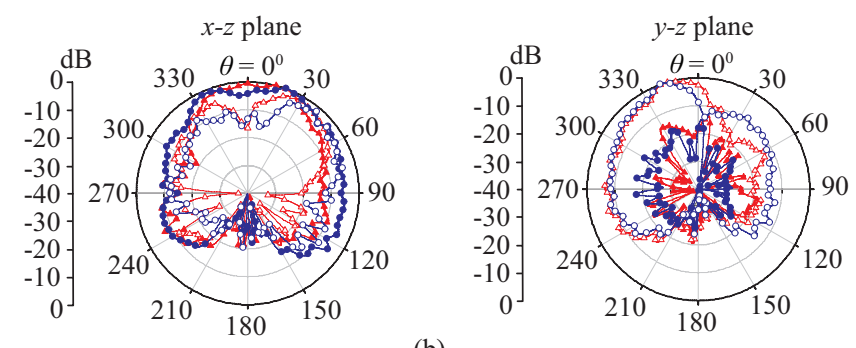

(b)

- Meas. $\mathrm{E}_{\phi}$ single-layer radiator $\multimap-$ Meas. $\mathrm{E}_{\theta}$ single-layer radiator $\neg$ Meas. $\mathrm{E}_{\phi}^{\phi}$ double-layer radiator $\longrightarrow-$ Meas. $\mathrm{E}_{\theta}$ double-layer radiator

Fig. 14. Measured normalized radiation patterns of the double-layer radiator antenna in free space along with that of the single-layer radiator antenna: (a) $2.5 \mathrm{GHz}$, (b) $5 \mathrm{GHz}$.

prototype with the double-layer radiator along with that with the single-layer radiator. It can be seen that the utilization of double-layer mesh indeed decreases the antenna transparency, particularly on the patch section, by approximately $20 \%$ based on the spectrophotometer measurement result shown in Fig. 1(b). However, this level of transparency is still sufficient for most applications.

After fabricating the second antenna its performance was measured and compared with the first antenna to investigate the effectiveness of using two layers of conductor as the radiating element. Fig. 13 shows the measured $\left|S_{11}\right|$ of the 


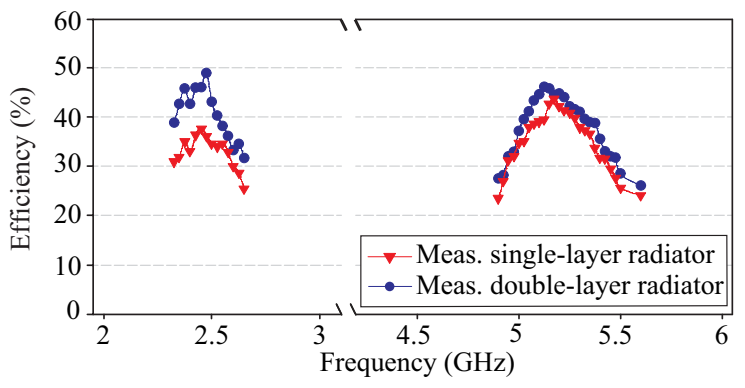

Fig. 15. Measured efficiency of the single-layer and double-layer radiator antennas over the operating bands.

double-layer radiator antenna compared with the measured result of the single-layer radiator antenna. The measured 10$\mathrm{dB}$ return-loss bandwidth of the double-layer radiator antenna is $260 \mathrm{MHz}$ (from $2.38 \mathrm{GHz}$ to $2.64 \mathrm{GHz}$ ) in the first band and $1,000 \mathrm{MHz}$ (from $4.4 \mathrm{GHz}$ to $5.4 \mathrm{GHz}$ ) in the second band.

Fig. 14 displays the measured far-field radiation patterns of the double-layer radiator antenna along with those of the single-layer radiator antenna in the $x-z$ and $y-z$ planes at 2.5 $\mathrm{GHz}$ and $5 \mathrm{GHz}$. They show that the radiation patterns of the double-layer radiator antenna are very similar to those of the single-layer radiator antenna; both antennas radiate best in the broadside direction. The maximum measured efficiency of the double-layer radiator antenna is $48 \%$ in the lower band and $46 \%$ in the higher band; these values were $37 \%$ and $44 \%$ for single-layer radiator antenna. The measured efficiencies of the double-layer radiator antenna and the singlelayer radiator antenna over the operating bands are illustrated in Fig. 15, which illustrates the efficiency improvement by using double-layer radiating element. The measured peak gain of the double-layer radiator antenna is $3.15 \mathrm{dBi}$ in the lower band and $3.53 \mathrm{dBi}$ in the higher band. For clarity, the performances of the antennas with single-layer and doublelayer mesh are summarized in Table III. Improvements in the gains and efficiencies of the antenna are obvious, particularly in the lower band. The improvement in the higher band seems to be offset by the loss of PDMS, which is approximately higher than the lower band (i.e., the loss tangent is double than that in the lower band). Nevertheless, these results validate the usefulness of the proposed technique in improving the performance of PDMS-embedded conductivemesh-based antennas by reducing the discontinuity in the conductive layer. One possibility we consider to be promising as future work to further increase the efficiency of the proposed antenna is the improvement of the effective conductivity of the conductive mesh through repetitive coating [25] of the mesh with suitable conductive materials without prejudicing the transparency. Another possibility would be to reduce the loss of PDMS by mixing it with micro/nanoparticles such as glass microspheres which has been successfully demonstrated by [26] in millimeter-wave region.

Finally, the overall antenna performance is compared with that of previously reported transparent antennas in Table IV. As can be seen, most of the antennas in the previous works are rigid and therefore not suitable for wearable applications
TABLE III

Summary OF THE PERFormanCES OF THE SINGLE-LAYER AND DOUBLE-LAYER RADIATOR ANTENNAS

\begin{tabular}{c|c|c|c|c|c|c}
\hline \multirow{2}{*}{ Band } & \multicolumn{2}{|c|}{ Bandwidth (MHz) } & \multicolumn{2}{c|}{ Peak Gain (dBi) } & \multicolumn{2}{c}{ Max. Eff. (\%) } \\
\cline { 2 - 7 } & SL & DL & SL & DL & SL & DL \\
\hline \hline Lower & 200 & 260 & 2.2 & 3.15 & 37 & 48 \\
Higher & 900 & 1000 & 3.02 & 3.53 & 44 & 46 \\
\hline
\end{tabular}

Note: SL: Single Layer, DL: Double Layer

which require flexibility and conformability. In terms of performance, the antenna realized with our proposed PDMSconductive-mesh composite is still comparable with some previously reported flexible as well as non-flexible transparent antennas. More importantly, the proposed approach, which combines commercially available transparent conductive mesh with solution-processable PDMS, allows for a transparent antenna realization process that is simple, effective, reproducible, and potentially scalable.

\section{CONCLUSION}

In this paper we have examined the physical characteristics and microwave performance of a conductive-mesh-polymer composite and have introduced a simple, efficient and inexpensive realization process to fabricate robust transparent wearable antenna. The measured performance of the dualband antenna validates the suitability of the proposed materials and realization process. The fabricated antenna is optically transparent, flexible, robust against physical deformation, has stable performance near a human body and has low SAR. The antenna retains good performance on human-body-equivalent phantoms and under various bending scenarios. Strong integration of the fine conductive mesh with PDMS is the main reason for its robustness against bending. The utilization of a full ground plane below the radiating patch shields the radiating patch from a lossy human body. The relatively lower gain and efficiency of the first antenna were improved by using a double-layer radiating element, which is a novel approach for improving the radiation performance of unconventionalmaterials based flexible antennas. Although the double-layer patch decreases the optical transparency, the antenna gain and efficiency were improved, especially in the lower band. The above-mentioned advantages make this antenna and its method of realization useful for wearable wireless systems and other applications that require flexible, durable and transparent antennas and microwave/radio-frequency circuits.

\section{ACKNOWLEDGMENT}

The authors would like to thank Dr Rajas Prakash Khokle for his assistance with antenna measurements and Elizabeth Valentin from The Ithree Institute, University of Technology, Sydney for taking the SEM image of the composite material.

\section{REFERENCES}

[1] P. S. Hall, Y. Hao, Y. I. Nechayev, A. Alomainy, C. C. Constantinou, C. Parini, M. R. Kamarudin, T. Z. Salim, D. T. M. Hee, R. Dubrovka, A. S. Owadally, W. Song, A. Serra, P. Nepa, M. Gallo, and M. Bozzetti, "Antennas and propagation for on-body communication systems," IEEE Antennas Propag. Mag., vol. 49, no. 3, pp. 41-58, June 2007. 
TABLE IV

Performance Comparison of the Proposed Antenna with Other State-OF-the-art Transparent Antennas

\begin{tabular}{c|c|c|c|c|c|c|c|c}
\hline Ant. & Conductor, Substrate & Flex. & $f_{c}(\mathrm{GHz})$ & FBW $(\%)$ & Max. Eff. $(\%)$ & Peak Gain $(\mathrm{dBi})$ & Transp. $(\%)$ & Size $\left(\lambda_{g}{ }^{3}\right)$ \\
\hline \hline$[5]$ & IZTO/Ag/IZTO film, Acryl & No & 2.45 & 0 & 7.7 & -4.23 & 68.6 & $0.67 \times 0.67 \times 0.013$ \\
{$[5]$} & Cu-mesh film, Acryl & No & 2.45 & 4 & 42.7 & 2.63 & 62.1 & $0.67 \times 0.67 \times 0.013$ \\
{$[6]$} & Cu mesh, Acrylic & No & 2.49 & 8 & 49.2 & 4.38 & 77.8 & $0.67 \times 0.67 \times 0.016$ \\
{$[7]$} & Ag grid, Corning glass & No & 2.03 & 19.7 & 60 & 5.5 & 78.3 & $4.85 \times 4.85 \times 0.32$ \\
{$[8]$} & ITO film, Quartz glass & No & 9.85 & 7.1 & 56.6 & 4.27 & NA & NA \\
{$[9]$} & ITO/Cu/ITO film, Corning glass & No & 0.8 & NA & NA & -1.96 & 61 & $0.94 \times 0.94 \times 0.44$ \\
{$[10]$} & FTO film, Pyrex glass & No & $2.5,5$ & 19,12 & NA & $0.43,3.63$ & 74.3 & NA \\
{$[12]$} & AgHT film, Glass & No & 2.05 & 34.1 & 68 & 0 & NA & $0.95 \times 0.95 \times 0.048$ \\
{$[13]$} & Gold/ITO film, Borosilicate glass & No & 9 & 155.6 & 70 & 3.5 & 85 & $2.39 \times 2.39 \times 0.06$ \\
{$[14]$} & IZTO/Ag/IZTO film, Polyimide & Yes & 2.45 & 16.3 & 34.8 & 2.89 & 81.1 & $0.77 \times 0.15 \times 0.002$ \\
{$[17]$} & Tortuous Cu micromesh, PDMS & Yes & 2.92 & 6.9 & 81.4 & -0.02 & 32 & $0.1 \times 0.14 \times 0.006$ \\
{$[19]$} & Ag mesh film, Corning glass & No & $8.8-10$ & 27 & NA & 3.8 & 82.6 & $2.5 \times 2.5 \times 0.034$ \\
Ours & Zn/Ni/Cu/Poly. mesh (SL), PDMS & Yes & $2.43,5.15$ & $8.2,17.5$ & 37,44 & $2.2,3.02$ & 72 & $0.67 \times 0.77 \times 0.06$ \\
Ours & Zn/Ni/Cu/Poly. mesh (DL), PDMS & Yes & $2.51,4.9$ & $10.4,20.4$ & 48,46 & $3.2,3.5$ & 52 & $0.67 \times 0.77 \times 0.06$ \\
\hline
\end{tabular}

Note: $f_{c}$ : Centre Frequency, SL: Single Layer, DL: Double layer

[2] P. Nepa and H. Rogier, "Wearable antennas for off-body radio Links at VHF and UHF bands: challenges, the state of the art, and future trends below 1 GHz," IEEE Antennas Propag. Mag., vol. 57, no. 5, pp. 30-52, Oct 2015.

[3] A. Tsolis, W. G. Whittow, A. A. Alexandridis, and J. C. Vardaxoglou, "Embroidery and related manufacturing techniques for wearable antennas: challenges and opportunities," Electronics, vol. 3, no. 2, pp. 314 338, 2014.

[4] G. Ginestet, N. Brechet, J. Torres, E. Moradi, L. Ukkonen, T. Bjrninen, and J. Virkki, "Embroidered Antenna-Microchip Interconnections and Contour Antennas in Passive UHF RFID Textile Tags," IEEE Antennas and Wireless Propagation Letters, vol. 16, pp. 1205-1208, 2017.

[5] S. Hong, Y. Kim, and C. W. Jung, "Transparent microstrip patch antennas with multilayer and metal-mesh films," IEEE Antennas and Wireless Propagation Letters, vol. 16, pp. 772-775, 2017.

[6] S. H. Kang and C. W. Jung, "Transparent patch antenna using metal mesh," IEEE Transactions on Antennas and Propagation, vol. 66, no. 4, pp. 2095-2100, April 2018.

[7] J. Hautcoeur, F. Colombel, M. Himdi, X. Castel, and E. M. Cruz, "Large and Optically Transparent Multilayer for Broadband H-Shaped Slot Antenna," IEEE Antennas and Wireless Propagation Letters, vol. 12, pp. 933-936, 2013.

[8] G. Sun, B. Muneer, and Q. Zhu, "A study of microstrip antenna made of transparent ITO films," in 2014 IEEE Antennas and Propagation Society International Symposium (APSURSI), July 2014, pp. 1867-1868.

[9] F. Colombel, X. Castel, M. Himdi, G. Legeay, S. Vigneron, and E. M. Cruz, "Ultrathin metal layer, ITO film and ITO/Cu/ITO multilayer towards transparent antenna," IET Sci., Measure. Tech., vol. 3, no. 3, pp. 229-234, May 2009.

[10] S. Sheikh, M. Shokooh-Saremi, and M. Bagheri-Mohagheghi, "Transparent microstrip patch antenna based on fluorine-doped tin oxide deposited by spray pyrolysis technique," IET Microwaves, Antennas Propagation, vol. 9, no. 11, pp. 1221-1229, 2015.

[11] M. E. Zamudio, T. Busani, Y. Tawk, J. Costantine, and C. Christodoulou, "Design of AZO film for optically transparent antennas," in Proc. IEEE Int. Symp. on Ant. and Propag. (APSURSI), June 2016, pp. 127-128.

[12] H. J. Song, T. Y. Hsu, D. F. Sievenpiper, H. P. Hsu, J. Schaffner, and E. Yasan, "A method for improving the efficiency of transparent film antennas," IEEE Antennas Wireless Propag. Lett., vol. 7, pp. 753-756, 2008.

[13] M. R. Haraty, M. Naser-Moghadasi, A. A. Lotfi-Neyestanak, and A. Nikfarjam, "Improving the efficiency of transparent antenna using gold nanolayer deposition," IEEE Antennas Wireless Propag. Lett., vol. 15, pp. 4-7, 2016.

[14] S. Hong, S. H. Kang, Y. Kim, and C. W. Jung, "Transparent and flexible antenna for wearable glasses applications," IEEE Trans. Antennas Propag., vol. 64, no. 7, pp. 2797-2804, July 2016.

[15] G. Clasen and R. Langley, "Meshed patch antennas," IEEE Trans. Antennas Propag., vol. 52, no. 6, pp. 1412-1416, June 2004.
[16] J. Hautcoeur, L. Talbi, K. Hettak, and M. Nedil, "60 GHz optically transparent microstrip antenna made of meshed AuGL material," IET Microw., Ant. and Propag., vol. 8, no. 13, pp. 1091-1096, October 2014.

[17] T. Jang, C. Zhang, H. Youn, J. Zhou, and L. J. Guo, "Semitransparent and flexible mechanically reconfigurable electrically small antennas based on tortuous metallic micromesh," IEEE Trans. Antennas Propag., vol. 65, no. 1, pp. 150-158, Jan 2017.

[18] J. Hautcoeur, F. Colombel, X. Castel, M. Himdi, and E. M. Cruz, "Radiofrequency performances of transparent ultra-wideband antennas," Prog. in Electromag. Res. C, vol. 22, pp. 259-271, 2011.

[19] A. Martin, X. Castel, O. Lafond, and M. Himdi, "Optically transparent frequency-agile antenna for X-band applications," Electronics Letters, vol. 51, no. 16, pp. 1231-1233, 2015.

[20] P. Bodo and J.-E. Sundgren, "Titanium deposition onto ion-bombarded and plasma-treated polydimethylsiloxane: Surface modification, interface and adhesion," Thin Solid Films, vol. 136, no. 1, pp. 147-159, 1986.

[21] R. B. V. B. Simorangkir, Y. Yang, L. Matekovits, and K. P. Esselle, "Dual-Band Dual-Mode Textile Antenna on PDMS Substrate for BodyCentric Communications," IEEE Antennas and Wireless Propagation Letters, vol. 16, pp. 677-680, 2017.

[22] Z. Xu, T. Kaufmann, and C. Fumeaux, "Wearable textile shielded stripline for broadband operation," IEEE Microw. Compon. Lett., vol. 24, no. 8, pp. 566-568, 2014.

[23] K. P. Latti, M. Kettunen, J. P. Strom, and P. Silventoinen, "A review of microstrip T-resonator method in determining the dielectric properties of printed circuit board materials," IEEE Trans. Instrum. Meas., vol. 56, no. 5, pp. 1845-1850, Oct 2007.

[24] K. Ito, K. Furuya, Y. Okano, and L. Hamada, "Development and characteristics of a biological tissueequivalent phantom for microwaves," Electronics and Communications in Japan (Part I: Communications), vol. 84, no. 4, pp. 67-77, Apr. 2001.

[25] Y. Bayram, Y. Zhou, B. S. Shim, S. Xu, J. Zhu, N. A. Kotov, and J. L. Volakis, "E-textile conductors and polymer composites for conformal lightweight antennas," IEEE Trans. Antennas Propag., vol. 58, no. 8, pp. 2732-2736, Aug 2010 .

[26] W. A. W. Muhamad, R. Ngah, M. F. Jamlos, P. J. Soh, and M. T. Ali, "High-gain dipole antenna using polydimethylsiloxane-glass microsphere (PDMS-GM) substrate for 5G applications," Appl. Physics A, vol. 123 , no. 1 , p. 102 , Dec 2016. 


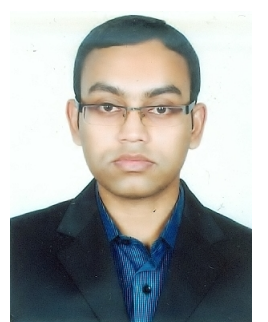

Abu Sadat Md. Sayem (S'17) received the BSc (First class Honours with first merit position) and MSc degree in Electrical and Electronic Engineering from the Rajshahi University of Engineering \& Technology, Bangladesh in 2011 and 2015 respectively. Currently he is pursuing the $\mathrm{PhD}$ degree in the School of Engineering, Macquarie University, Sydney, NSW 2109, Australia.

$\mathrm{Mr}$ Sayem worked as an assistant professor in the department of electrical and electronic engineering in the Rajshahi University of Engineering \& Technology, Bangladesh from 2015 to 2017 (now on study leave to pursue PhD) and as a lecturer in the same department from 2012 to 2015 . He is a graduate student member of IEEE from 2017. His current research interests include wearable antennas, flexible antennas, transparent antennas and antennas for biomedical applications.

Mr Sayem is a recipient of several prestigious awards and scholarships, including the Commonwealth Government funded International Research Training Program Scholarship to support the $\mathrm{PhD}$ in Australia, the Prime Minister Gold Medal from Bangladesh and the University Gold Medal from Rajshahi University of Engineering \& Technology, Bangladesh.

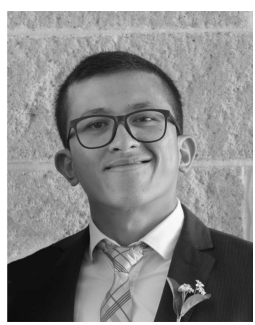

Roy B. V. B. Simorangkir (S'15-M'18) received the BS degree in Telecommunication Engineering from the Bandung Institute of Technology, Bandung, Indonesia in 2010, the MS degree in Electrical and Electronic Engineering from Yonsei University, Seoul, South Korea in 2014, and the PhD degree in Electronic Engineering from Macquarie University, Sydney, Australia in 2018.

$\mathrm{He}$ held a teaching staff position at Institut Teknologi Harapan Bangsa, Bandung, Indonesia from August 2010 to August 2011 and at Macquarie University International College from March 2018 to May 2019. He was also with the Reconfigurable Electronics and Antennas Research Group as a casual research officer from September 2018 to December 2018. Currently, he is a post-doctoral research associate at the Institute of Electronics and Telecommunications of Rennes (IETR), University of Rennes 1 . His general research interests include design and fabrication of flexible wearable antennas, reconfigurable antennas, ultra-wideband (UWB) antennas, leaky wave antennas (LWAs), and flexible wearable sensors.

He was selected as a finalist in the Student Paper and Advanced Practice Paper Competitions of the 2017 IEEE MTT-S International Microwave Symposium (IMS), Honolulu, HI. He also received the First Prize in the 2017 IEEE Region 10 Student Paper Contest, postgraduate category.

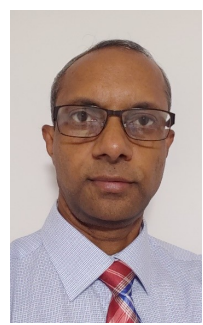

Professor Karu P. Esselle, IEEE M (1992), SM (1996), F (2016), received BSc degree in electronic and telecommunication engineering with First Class Honours from the University of Moratuwa, Sri Lanka, and MASc and PhD degrees with nearperfect GPA in electrical engineering from the University of Ottawa, Canada. He is a Professor of Electronic Engineering, Macquarie University, Sydney, Past Director of WiMed Research Centre and Past Associate Dean Higher Degree Research (HDR) of the Division of Information and Communication Sciences. He has also served as a member of the Deans Advisory Council and the Division Executive and as the Head of the Department several times. In 2018, he has been selected to chair the prestigious Distinguished Lecturer Program Committee of the IEEE Antennas and Propagation (AP) Society the premier global learned society dedicated for antennas and propagation - which has over 8,000 members worldwide, and was re-selected in 2019. After two stages in the selection process, Karu was also selected by this Society as one of two candidates in the ballot for 2019 President of the Society. Only three people from Asia or Pacific apparently have received this honour (one from Australia and two from Japan) in the 68-year history of this Society. Karu is also one of the three Distinguished Lecturers (DL) selected by the Society in 2016. He is the only Australian to chair the AP DL Program ever, the only Australian AP DL in almost two decades, and second Australian AP DL ever. He has been continuously serving the IEEE AP Society Administrative Committee in several elected or ex-officio positions since 2015. Karu is also the Chair of the Board of management of Australian Antenna Measurement Facility, and was the elected Chair of both IEEE New South Wales (NSW), and IEEE NSW AP/MTT Chapter, in 2016 and 2017. He directs the Centre for Collaboration in Electromagnetic and Antenna Engineering. Karu was elevated to prestigious IEEE Fellow grade for his contributions to resonancebased antennas. He is also a Fellow of Engineers Australia.

Karu has authored approximately 600 research publications and his papers have been cited about 8,000 times. He is the first Australian antenna researcher ever to reach Google Scholar h-index of 30 and his citation indices have been among the top Australian antenna researchers for a long time i10 index (in June 2019: i10 is 158 and h-index is 42). Since 2002, his research team has been involved with research grants, contracts and $\mathrm{PhD}$ scholarships worth over 18 million dollars, including 15 Australian Research Council grants, without counting the 245 million-dollar SmartSat Corporative Research Centre, which started in 2019. His research has been supported by many national and international organisations including Australian Research Council, Intel, US Air Force, Cisco Systems, Hewlett-Packard, Australian Department of Defence, Australian Department of industry, and German and Indian governments.

Karu is in the College of Expert Reviewers of the European Science Foundation (2019-22) and he has been invited to serve as an international expert/research grant assessor by several other research funding bodies as well, including European Research Council and national agencies in the Netherlands, Canada, Finland, Hong-Kong, Georgia, South Africa and Chile. He has been invited by Vice-Chancellors of Australian and overseas universities to assess applications for promotion to professorial levels. He has also been invited to assess grant applications submitted to Australia's most prestigious schemes such as Australian Federation Fellowships and Australian Laureate Fellowships. In addition to the large number of invited conference speeches he has given, he has been an invited keynote speaker of several IEEE and URSI conferences and workshops.

Karu's awards include 2019 Motohisa Kanda Award for the most cited paper in IEEE Transactions EMC in the past five years, 2019 ARC Discovery International Award, 2017 Excellence in Research Award from the Faculty of Science and Engineering, 2017 Engineering Excellence Award for Best Innovation, 2017 Highly Commended Research Excellence Award from Macquarie University, 2017 Certificate of Recognition from IEEE Region 10, 2016 and 2012 Engineering Excellence Awards for Best Published Paper from IESL NSW Chapter, 2011 Outstanding Branch Counsellor Award from IEEE headquarters (USA), 2009 Vice Chancellor's Award for Excellence in Higher Degree Research Supervision and 2004 Innovation Award for best invention disclosure. His mentees have been awarded many fellowships, awards and prizes for their research achievements. Forty-eight international experts who examined the theses of his recent $\mathrm{PhD}$ graduates ranked them in the top 5\% or $10 \%$.

Karu has provided expert assistance to more than a dozen companies including Intel, Hewlett Packard Laboratory (USA), Cisco Systems (USA), Audacy (USA), Cochlear, Optus, ResMed and Katherine-Werke (Germany). $\mathrm{He}$ is an Associate Editor of IEEE Transactions on Antennas Propagation as well as IEEE Access.

Karu is the Technical Program Committee Co-Chair of ISAP 2015, APMC 2011 and TENCON 2013 and the Publicity Chair of ICEAA 2016, IWAT 
2014 and APMC 2000. His research activities are posted in the web at http://web.science.mq.edu.au/ esselle/ .

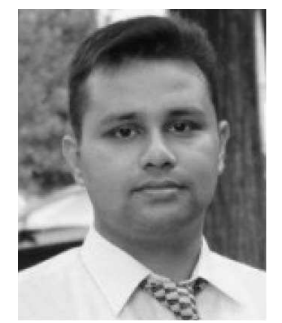

Raheel M. Hashmi (S'12-M'15) received the BS degree (Hons I) from the COMSATS Institute of Information Technology, Islamabad, Pakistan, in 2009, the MS degree from the Politecnico di Milano, Milan, Italy, in 2011, and the $\mathrm{PhD}$ degree from Macquarie University, Sydney, NSW, Australia, in 2015.

He held a research associate position at the Reconfigurable Electronics and Antennas Research Group, Macquarie University. He is currently a Lecturer with the School of Engineering, Macquarie University. He has authored over 40 refereed journal and conference publications. He is an Inventor of two patent applications. His current research interests include EBG structures and their use in electromagnetic applications, EBG resonator antennas, FSS, wideband directive antennas, ultrawideband antennas, and antennas for biomedical applications.

Dr Hashmi was a recipient of several prestigious awards and fellowships, including the URSI Young Scientist Award, the Commonwealth International Postgraduate Research Scholarship, the CSIRO OCE PhD Fellowship, and the Institutes Gold Medal from the COMSATS Institute of Information Technology. He is the Chair of the IEEE Young Professionals Affinity Group, NSW, and the Secretary of the IEEE Antennas and Propagation/MTT Joint Chapter, NSW. He has been serving as an Associate Editor of IET Microwaves, Antennas and Propagation since 2016. 